\title{
Modelos predictivos de la producción de Pinus taeda empleando variables vinculadas con las podas
}

\author{
Yield prediction models using pruning variables for Pinus taeda
}

\author{
Rubén Costas $^{1 *}$, Patricio Mac Donagh ${ }^{1}$, Elizabeth Weber ${ }^{1}$, Santiago Figueredo ${ }^{1}$, \\ Claudio Gómez ${ }^{1}$, Pedro Irschick ${ }^{1}$
}

\begin{abstract}
*Autor de correspondencia: ${ }^{1}$ Universidad Nacional de Misiones, Facultad de Ciencias Forestales, Bertoni 124, Eldorado (3380), Misiones, Argentina, Tel.: 54-3751-431526, Fax 54-3751-431766, rcostas@ facfor.unam.edu.ar
\end{abstract}

\begin{abstract}
SUMMARY
Based on data from an experience in a Pinus taeda plantation, including ages between 4 and 7 years old, multiple linear regressions were adjusted to obtain stand yield functions including regression variables associated with prunes intensity and opportunity, besides age, stand density and site index. The response variables analyzed were quadratic mean diameter, basal area and volume per hectare. The results show that it was possible to improve the prediction quality and accuracy of models of basal area when prune variables are considered, with respect to equations without these variables and with statistical significance. Improvements of $9.19 \%$ of the standard residual error and $8.99 \%$ of the statistics indicator employed to compare the prediction capacity were obtained. The utilization of some of those functions like sub-models inside equations of quadratic mean diameter and volume by hectare, simple and with good statistics, gives results that are sensitive to prune. These functions could be employed in spreadsheets to decide when and how many green branches to prune in relation to the production objective. The obtained models appear interesting for developing new local growth simulation programs of Pinus taeda that include prunes applied in intensive silvicultural systems of forest production.
\end{abstract}

Key words: Pinus taeda, yield models, crown ratio, quadratic mean diameter, volume per hectare.

\section{RESUMEN}

Con datos de un ensayo de Pinus taeda, con edades entre 4 y 7 años, se ajustaron a través de regresión lineal múltiple, modelos de producción estáticos de rodal completo, incluyendo variables regresoras asociadas con la intensidad y oportunidad de podas, además de la edad, la densidad y el índice de sitio. Las variables respuestas analizadas fueron el diámetro medio cuadrático, el área basal y el volumen por hectárea. Respecto del área basal fue posible obtener modelos sensibles a las podas realizadas, de mejor calidad de ajuste y de capacidad de predicción que ecuaciones sin variables vinculadas con las podas, que tuvieron excelentes comportamientos estadísticos. Se lograron mejoras de hasta 9,19\% de error estándar residual y de 8,99\% del estadístico usado para comparar la capacidad de predicción. La utilización de algunas de esas funciones como submodelos dentro de ecuaciones predictivas de diámetro medio cuadrático y volumen por hectárea, relativamente sencillos y con confiabilidad estadística, produjo resultados sensibles a las podas realizadas. Las funciones obtenidas pueden ser usadas como herramientas de cálculo para decidir cuánto y cuándo podar ramas fotosintéticamente activas de acuerdo a los objetivos de producción. Estos modelos son promisorios para el desarrollo de nuevos programas locales de simulación de crecimiento y producción regionales de $P$. taeda que contemplen a las podas realizadas en los sistemas de producción intensivos.

Palabras clave: Pinus taeda, modelos de producción, proporción de copa, diámetro medio cuadrático, volumen por hectárea.

\section{INTRODUCCIÓN}

La producción de una masa forestal regular es predecible a través de modelos matemáticos. Las variables predictoras más usadas suelen ser la edad, la densidad y la calidad de sitio expresada a través del índice de sitio o la altura dominante a una edad determinada. Esos modelos son tratados por Alder (1980), Avery y Burkhart (1994), Sanquetta (1996), Prodan et al. (1997), Crechi et al. (1998) y Crechi et al. (1999), entre otros autores.
En la provincia de Misiones y noreste de Corrientes, Argentina, en los últimos quince años, se han difundido e implementado sistemas intensivos de manejo silvícola de plantaciones de coníferas. El control de la densidad y realización de podas para optimizar la cantidad y calidad de la producción forestal han mostrado ser herramientas eficaces para satisfacer las demandas del mercado local e internacional. 
La poda de ramas vivas reduce el área fotosintética de la copa de los árboles, produciendo reducción del crecimiento de los mismos. Evans (1992), Seitz (1995) citan experiencias realizadas con Pinus sp., en las cuales observan que, a mayor proporción de copa extraída, la disminución del crecimiento es más drástica. Costas et al. (2005), en un ensayo de Pinus taeda L. con control de densidad y podas, encuentran que los diámetros medios y los volúmenes totales por hectárea producidos con alturas de poda finales de $3,3 \mathrm{~m}$, logradas con tres y cuatro levantes, resultan significativamente superiores a los observados con alturas de poda finales de $6,6 \mathrm{~m}$, logradas con dos y tres levantes. Como la interacción entre la densidad y las podas de ramas vivas no resultó significativa $(P<0,05)$, concluyen que ambos factores produjeron efectos independientemente uno de otro, de manera similar a lo encontrado por Muñoz et al. (2005), quienes evalúan un ensayo factorial de raleo y poda en Eucalyptus nitens Maiden, con podas practicadas a una edad en que sólo una reducida porción del fuste podado hasta 7 $\mathrm{m}$ de altura presentaba ramas verdes. Meneses y Guzmán (2000), al analizar una muestra de 30 rodales de $P$. radiata con distinta historia de raleos y podas, señalan que podas muy severas influyen negativamente sobre el crecimiento diamétrico hasta la edad de cosecha y que el éxito de las podas depende de la calidad de sitio y de la oportunidad e intensidad de las mismas.

Existen modelos de crecimiento diamétrico como los ajustados por Burkhart et al. (2001) y Burkhart et al. (2003) que contienen entre sus variables regresoras a la proporción de longitud de copa viva respecto de la altura del árbol (CR). Las funciones presentadas en esos trabajos indican que con el aumento de CR se incrementa el crecimiento del diámetro a la altura de pecho (dap), reflejando el mayor potencial de fotosíntesis que tienen plantas con mayor proporción de longitud de copa respecto de la altura del árbol.

La combinación de factores biométricos y económicos relacionados con el costo de la realización de podas y la ganancia en valor de la producción por efectos de las mismas, justifican la investigación sobre modelos que reflejen su influencia sobre la producción de plantaciones de coníferas en el noreste argentino. Los antecedentes señalan que la realización de podas resulta onerosa (Evans 1992), con una incidencia entre 10 y $20 \%$ respecto del costo total de actividades realizadas en plantaciones locales de $P$. taeda hasta el momento de cosecha. Durante el trienio 2003-2005, en la zona norte de Misiones, Argentina, el precio de trozas de esta especie con podas tempranas fue aproximadamente $40 \%$ superior al pagado por trozas sin podas para categorías similares de punta fina.

El desarrollo de modelos "sensibles" a las podas sería de gran utilidad en la planificación y en la gestión forestal. Esas funciones deberían ser biológicamente razonables (Huang et al. 2003) y más eficientes que las dispo- nibles para esta especie en la región (Crechi et al. 1999), que predicen la producción en función únicamente de la edad, la densidad y la calidad de sitio. Basados en la hipótesis que la incorporación de variables asociadas a las podas producen mejoras estadísticas en la predicción del rendimiento, este trabajo tuvo los siguientes objetivos: a) generar modelos de producción estáticos de rodal completo, que tengan entre sus variables predictoras a las relacionadas con las podas, para edades entre cuatro y siete años, y b) evaluar y analizar la mejora en calidad de ajuste y capacidad de predicción de dichos modelos respecto de funciones sin variables independientes vinculadas con las podas.

\section{MÉTODOS}

El material experimental es un ensayo generado a partir de una plantación coetánea de $P$. taeda procedencia Marion, implantada en 1996 en la localidad de Garuhapé-mí, Departamento Libertador General San Martín, Misiones. La plantación original fue realizada con una densidad de 2.222 plantas $\mathrm{ha}^{-1}$, sobre un suelo no compactado correspondiente a un Kandiudult típico (Soil Survey Staff 1991), con una pendiente máxima menor al $3 \%$. En la preparación del terreno se utilizó rastra de discos. No se aplicaron fertilizantes en ningún momento previo o posterior al establecimiento de la plantación. El control de malezas fue realizado combinando aplicación de herbicidas, desmalezado manual y mecánico a través del paso de rastra de discos. La sobrevivencia media de plantas en los dos primeros años de plantación fue de $80 \%$, con pérdidas de plantas debidas al ataque de hormigas, falta de agua y/o plantación defectuosa. A los tres años de edad se realizó una poda de aproximadamente $1,8 \mathrm{~m}$ de altura sobre toda la masa y sobre dos tercios de la plantación fueron realizados raleos sistemáticos. En uno de ellos se eliminó el 33,3\% y en el otro el $66,7 \%$ de las plantas.

El ensayo tiene una superficie total de $19.080 \mathrm{~m}^{2}$. Consistió en un experimento factorial con 4 alturas finales de poda, tres niveles diferentes de número de levantes de poda y tres densidades manejadas con raleos por lo bajo. El criterio adoptado para la realización de las podas en cada oportunidad fue con altura de poda homogénea en todos los árboles de cada unidad experimental (Seitz 1995) (cuadro 1). Cada una de las 36 combinaciones de densidad y tratamientos de poda tuvo dos parcelas como repeticiones. Las alturas medias a los 4,5 y 6 años de edad fueron 7,2, 9,3 y 10,8 $\mathrm{m}$ y los porcentajes máximos de copa removida, derivados de la aplicación de los tratamientos de poda más drásticos en cada una de esas edades, fueron 47,9, 64,1 y 35,6\% respectivamente. En todas los casos fueron podadas ramas vivas, excepto en la última poda efectuada en las parcelas con mayor densidad donde se aplicó el tratamiento de poda 12, en el 
cual aproximadamente el $50 \%$ de las ramas eliminadas estaban muertas.

Desde el $4^{\circ}$ hasta el $6^{\circ}$ año de edad se midieron los dap, las alturas de poda (hp) y las alturas totales (ht) de todos los árboles de las 72 unidades experimentales. A la edad de 7 años se repitió la metodología de medición de diámetros y se midieron las ht de sólo cuatro árboles por parcela: el de mayor dap, el de menor dap y dos con dap medianos dentro de la parcela. El resto de las ht fueron estimadas a través de una relación hipsométrica ajustada con los 288 pares de valores $\left(\mathrm{dap}_{\mathrm{i}} \mathrm{ht}_{\mathrm{i}}\right)$ recolectados. Con los registros obtenidos y las funciones correspondientes, se calcularon, para cada unidad experimental y a la edad respectiva, diámetro medio cuadrático $(\mathrm{Dg})$, área basal

Cuadro 1. Diseño factorial de tratamientos de densidad, número de levantes y altura de poda.

Factorial design for density, number of raise and prune height treatments.

\begin{tabular}{|c|c|c|c|c|c|}
\hline \multirow{2}{*}{ Densidad $^{\mathrm{a}}$} & \multirow{2}{*}{ Número de levantes de poda } & \multicolumn{3}{|c|}{ Altura de poda (m) } & \multirow{2}{*}{$\begin{array}{r}\text { Tratamie } \\
\text { de podi }\end{array}$} \\
\hline & & año 4 HP4 & año 5 HP5 & año 6 HP6 & \\
\hline \multirow{12}{*}{$\begin{array}{l}\mathrm{D}_{4}=567 \\
\mathrm{D} 1_{5}=525 \\
\mathrm{D} 1_{6}=499 \\
\mathrm{D}_{7}=400\end{array}$} & 2 & 3,30 & 3,30 & 3,3 & 1 \\
\hline & 3 & 2,60 & 3,30 & 3,3 & 2 \\
\hline & 4 & 2,37 & 2,83 & 3,3 & 3 \\
\hline & 2 & 4,40 & 4,40 & 4,4 & 4 \\
\hline & 3 & 3,15 & 4,40 & 4,4 & 5 \\
\hline & 4 & 2,73 & 3,56 & 4,4 & 6 \\
\hline & 2 & 1,80 & 5,50 & 5,5 & 7 \\
\hline & 3 & 3,70 & 3,70 & 5,5 & 8 \\
\hline & 4 & 3,10 & 4,30 & 5,5 & 9 \\
\hline & 2 & 1,80 & 6,60 & 6,6 & 10 \\
\hline & 3 & 4,25 & 4,25 & 6,6 & 11 \\
\hline & 4 & 3,47 & 5,03 & 6,6 & 12 \\
\hline \multirow{12}{*}{$\begin{array}{l}\mathrm{D} 2_{4}=810 \\
\mathrm{D} 2_{5}=760 \\
\mathrm{D} 2_{6}=760 \\
\mathrm{D} 2_{7}=700\end{array}$} & 2 & 3,30 & 3,30 & 3,3 & 1 \\
\hline & 3 & 2,60 & 3,30 & 3,3 & 2 \\
\hline & 4 & 2,37 & 2,83 & 3,3 & 3 \\
\hline & 2 & 4,40 & 4,40 & 4,4 & 4 \\
\hline & 3 & 3,15 & 4,40 & 4,4 & 5 \\
\hline & 4 & 2,73 & 3,56 & 4,4 & 6 \\
\hline & 2 & 1,80 & 5,50 & 5,5 & 7 \\
\hline & 3 & 3,70 & 3,70 & 5,5 & 8 \\
\hline & 4 & 3,10 & 4,30 & 5,5 & 9 \\
\hline & 2 & 1,80 & 6,60 & 6,6 & 10 \\
\hline & 3 & 4,25 & 4,25 & 6,6 & 11 \\
\hline & 4 & 3,47 & 5,03 & 6,6 & 12 \\
\hline \multirow{12}{*}{$\begin{array}{l}\mathrm{D} 3_{4}=1691 \\
\mathrm{D} 3_{5}=1480 \\
\mathrm{D} 3_{6}=1307 \\
\mathrm{D} 3_{7}=1000\end{array}$} & 2 & 3,30 & 3,30 & 3,3 & 1 \\
\hline & 3 & 2,60 & 3,30 & 3,3 & 2 \\
\hline & 4 & 2,37 & 2,83 & 3,3 & 3 \\
\hline & 2 & 4,40 & 4,40 & 4,4 & 4 \\
\hline & 3 & 3,15 & 4,40 & 4,4 & 5 \\
\hline & 4 & 2,73 & 3,56 & 4,4 & 6 \\
\hline & 2 & 1,80 & 5,50 & 5,5 & 7 \\
\hline & 3 & 3,70 & 3,70 & 5,5 & 8 \\
\hline & 4 & 3,10 & 4,30 & 5,5 & 9 \\
\hline & 2 & 1,80 & 6,60 & 6,6 & 10 \\
\hline & 3 & 4,25 & 4,25 & 6,6 & 11 \\
\hline & 4 & 3,47 & 5,03 & 6,6 & 12 \\
\hline
\end{tabular}

a $\quad \mathrm{D1}_{4}, \mathrm{D} 1_{5}, \ldots ., \mathrm{D} 3_{7}=$ número de árboles por hectárea en cada nivel de densidad a cada edad. 
(G), altura dominante (Hd), obtenida del promedio de altura de la proporción de árboles que en cada parcela equivalen a los 100 árboles más gruesos por ha, volumen individual sin corteza y volumen total sin corteza por ha (V). Además, se calculó para cada árbol la proporción de longitud de copa respecto de la altura de fuste total $\left(\mathrm{cr}_{\mathrm{i}}\right)$, antes y después de realizadas las podas en cada año de edad, calculada por medio de la expresión $\mathrm{cr}_{\mathrm{i}}=\left(\mathrm{ht}_{\mathrm{i}}-\mathrm{hp}_{\mathrm{i}}\right) / \mathrm{ht}_{\mathrm{i}}$. Del promedio de los $c r_{\mathrm{i}}$ se obtuvo el CR para cada parcela, que fue la medida utilizada en el análisis posterior (cuadro 2).

Con la información de las variables obtenidas, algunas transformaciones y combinaciones entre ellas, se ajustaron funciones lineales predictoras del diámetro medio cuadrático (Dg), del área basal $(\mathrm{G})$ y del volumen por hectárea (V). Los modelos ajustados fueron generados por los autores, incorporando en una primera etapa variables vinculadas con las podas a modelos de producción existentes, y luego se exploró el desarrollo de otras funciones. En los ajustes, evaluación y selección de modelos se adoptó en general la metodología descrita en Myers (1990).

Previo a la utilización de la Hd y del índice de sitio (IS), calculado para cada par de valores $\left(\mathrm{Hd}_{\mathrm{i}}, \mathrm{Edad}_{\mathrm{i}}\right)$ como variables independientes, se realizaron análisis exhaustivos de sus comportamientos. Por un lado, la prueba $\mathrm{F}$ con un nivel de significación del $5 \%$, indicó que no hubo diferencias significativas de las $\mathrm{Hd}$ entre los tratamientos aplicados en ninguno de los años de medición. Por otro lado, los gráficos de diagnóstico señalaron que en cada edad los mayores Dg, G y V se produjeron en las parcelas con mayor Hd. Finalmente, se optó por utilizar a Hd e IS, ya que ambos reflejan las diferencias microam- bientales presentes en el ensayo, debidas probablemente a la acción antrópica efectuada sobre la superficie del ensayo previo al establecimiento de la plantación. Como, además, el IS fue calculado utilizando las ecuaciones de índice de sitio regionales de $P$. taeda presentadas por Crechi et al. (1999), las funciones de producción resultantes podrían ser usadas de manera compatible con aquellas.

Las ecuaciones predictoras de las variables respuestas analizadas fueron ajustadas a los datos a través de regresión lineal múltiple, usando el método de mínimos cuadrados para modelos lineales. Para comparar la calidad de ajuste de las funciones obtenidas se utilizaron el coeficiente de determinación ajustado ( $\mathrm{R}^{2}$ ajustado) y el error estándar de estimación $\left(\mathrm{S}_{\mathrm{yx}}\right)$. Además de la obtención de estimaciones de los coeficientes de regresión de cada modelo, en cada caso se utilizó la prueba t $(P<0,05)$ para determinar la significancia estadística de la contribución de cada término en la predicción de cada variable respuesta. Para comparar la capacidad predictora de los modelos obtenidos se realizaron validaciones de cada uno a través de la raíz cuadrada del error de predicción cuadrático medio (PRESS RMSE). Para obtener esta medida primero se calculó el estadístico PRESS, descrito por Myers (1991) y mencionado por Huang et al. (2003) como equivalente a la validación con una parte de la muestra usada en los ajustes y otra parte utilizada para validar a las funciones obtenidas. El PRESS de cada modelo se obtuvo de la suma de los cuadrados de los residuales $e_{i,-i}(i=1,2, \ldots n)$. Como en los ajustes de los modelos no siempre se utilizó la misma cantidad de observaciones (n), dependiendo de las variables regresoras presentes en cada función, se dividió el PRESS de cada una por n. Finalmente, se calculó la raíz cuadrada de ese cociente para obtener el estadístico PRESS

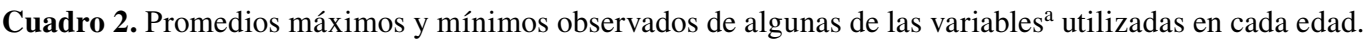
Average, minimum and maximum of some variables ${ }^{\mathrm{a}}$ used at each Age.

\begin{tabular}{ccccccrr}
\hline $\begin{array}{c}\text { Edad } \\
\text { (años) }\end{array}$ & Parámetro & $\mathrm{Dg}(\mathrm{cm})$ & $\mathrm{Hm}(\mathrm{m})$ & $\mathrm{Hd}(\mathrm{m})$ & $\mathrm{CR}$ & $\mathrm{G}\left(\mathrm{m}^{2} \mathrm{ha}^{-1}\right)$ & $\mathrm{V}\left(\mathrm{m}^{3} \mathrm{ha}^{-1}\right)$ \\
\hline \multirow{4}{*}{4} & Promedio & 10,51 & 7,21 & 7,99 & 0,74 & 8,48 & 24,23 \\
& Mínimo & 7,63 & 6,03 & 6,56 & 0,68 & 2,49 & 5,30 \\
& Máximo & 14,39 & 8,69 & 8,78 & 0,80 & 16,65 & 50,99 \\
\hline \multirow{2}{*}{5} & Promedio & 13,72 & 9,21 & 9,94 & 0,66 & 14,12 & 53,13 \\
& Mínimo & 9,75 & 7,63 & 8,62 & 0,52 & 5,50 & 17,25 \\
& Máximo & 18,36 & 10,63 & 11,19 & 0,83 & 26,77 & 107,34 \\
\hline \multirow{2}{*}{6} & Promedio & 16,69 & 11,05 & 11,66 & 0,62 & 18,12 & 78,56 \\
& Mínimo & 13,53 & 9,16 & 9,67 & 0,36 & 8,24 & 30,71 \\
& Máximo & 23,28 & 12,40 & 13,14 & 0,77 & 32,48 & 141,47 \\
\hline \multirow{2}{*}{7} & Promedio & 19,55 & 12,54 & 13,32 & 0,60 & 20,25 & 99,77 \\
& Mínimo & 15,56 & 11,09 & 11,75 & 0,44 & 9,93 & 46,24 \\
& Máximo & 27,36 & 13,92 & 15,00 & 0,76 & 32,58 & 166,14 \\
\hline
\end{tabular}

a $\quad \mathrm{Dg}=$ diámetro medio cuadrático; $\mathrm{Hm}=$ altura media; $\mathrm{Hd}=$ altura dominante; $\mathrm{CR}$ = promedio de proporción de longitud de copa respecto de la altura total de los árboles de cada unidad experimental; $\mathrm{G}=$ área basal; $\mathrm{V}=$ volumen total por hectárea. 
RMSE, expresado en las mismas unidades que cada variable respuesta. Al ajustar funciones donde la variable respuesta fue el logaritmo del área basal, los estadísticos $\mathrm{S}_{\mathrm{yx}}$ y PRESS RMSE fueron obtenidos con los residuales expresados en la unidad de medida natural de dicha variable $\left(\mathrm{m}^{2} \mathrm{ha}^{-1}\right)$. En todos los ajustes se comprobó el cumplimiento de los supuestos de homogeneidad de variancia y de distribución normal de los residuos. Las variables regresoras utilizadas fueron:

CR4: proporción de longitud de copa remanente promedio luego de las podas realizadas a los cuatro años de edad.

CR5: proporción de longitud de copa remanente promedio luego de las podas realizadas a los cinco años de edad.

CRA: proporción de longitud de copa remanente promedio a la edad de predicción.

CRM: promedio entre las proporciones de longitud de copa remanente desde la edad de cuatro años y hasta un año anterior a la edad de predicción, previas a las podas realizadas en cada oportunidad.

DCR4: diferencia de proporciones de copa remanentes entre la edad de predicción y los cuatro años de edad (CRA-CR4).

DHP4: diferencia entre las alturas de poda de la edad de predicción y la realizada a los cuatro años de edad (HPA-HP4).

E: $\quad$ edad de la masa (años).

EUP: edad de la masa al momento de realización de la última poda (años).

G: área basal presente a la edad de predicción $\left(\mathrm{m}^{2} \mathrm{ha}^{-1}\right)$

Hd: altura dominante a la edad de predicción (m).

HP4: altura de poda realizada en el $4^{\circ}$ año de edad (m).

HP5: altura de poda realizada en el $5^{\circ}$ año de edad (m).

HPA: altura de poda lograda por lo menos un año antes de la edad de predicción (m).

IS: $\quad$ índice de sitio (m).

$\mathrm{N}$ : número de árboles por hectárea registrado por lo menos un año antes de la edad de predicción.

NLP: número de levantes de poda realizados hasta llegar a la altura de poda actual.

\section{RESULTADOS}

Predicción del área basal. En el cuadro 3 se muestran las ecuaciones resultantes del ajuste de diez modelos lineales y algunos estadísticos indicadores de la calidad de ajuste y de la capacidad de predicción de cada uno. La función identificada con el número 1 fue la única que no contuvo a ninguna variable de poda. Todos los términos independientes de los modelos resultaron estadísticamente significativos. Del ajuste de las nueve funciones que contuvieron variables de poda, resultó que todas tuvieron menores $S_{\mathrm{yx}}$ y PRESS RMSE que la función 1. Teniendo en cuenta esos dos indicadores y el $\mathrm{R}^{2}$ ajustado, se destacaron las funciones 2 y 6 , por su calidad de ajuste, capacidad de predicción y porque en su aplicación se necesita sólo un término más que en la ecuación 1. Los ajustes de esos modelos produjeron mejoras de 6,98 y $9,19 \%$ en $\mathrm{S}_{\mathrm{yx}}$ y de 6,75 y 8,99\% en PRESS RMSE respectivamente, respecto de los resultados obtenidos al ajustar el modelo 1. Difieren en el tipo de información respecto de las podas que se necesitan para usar cada modelo, con connotaciones estadísticas y prácticas. Para utilizar la función 2 alcanza con calcular el CRA, que no necesariamente refleja toda la historia de podas aplicadas desde los 3 años hasta la edad de predicción y tuvo un coeficiente de correlación con $\mathrm{G}$ más débil que la variable CRM, presente en la ecuación 6. Para usar esta variable, que representa un promedio de la historia de las podas realizadas desde los tres años, se requiere un conocimiento preciso de la misma. En la figura 1 se muestran los valores de $\mathrm{G}$ predichos a una edad de siete años por el modelo 6 , considerando un IS $=23 \mathrm{~m}$. Puede observarse que por cada un aumento de 0,2 unidades de CRM, se produjo una disminución del área basal de $2,1 \mathrm{~m}^{2} \mathrm{ha}^{-1}$.

En todos los casos la presencia en esas funciones de las variables CR4, CRA, CRM y HPA como variables regresoras resultó ser estadísticamente significativa $(P<0,05)$. El área basal predicha fue mayor al incrementarse CR4, CRA y/o CRM y fue menor al aumentar HPA. No resultaron significativos los aportes de las variables NLP y EUP toda vez que fueron incorporadas como variables independientes en modelos ajustados no incluidos en el cuadro 3. Los modelos que tuvieron como variables regresoras a $\mathrm{CR} 4$ son utilizables para edades comprendidas desde los cinco y hasta los siete años inclusive.

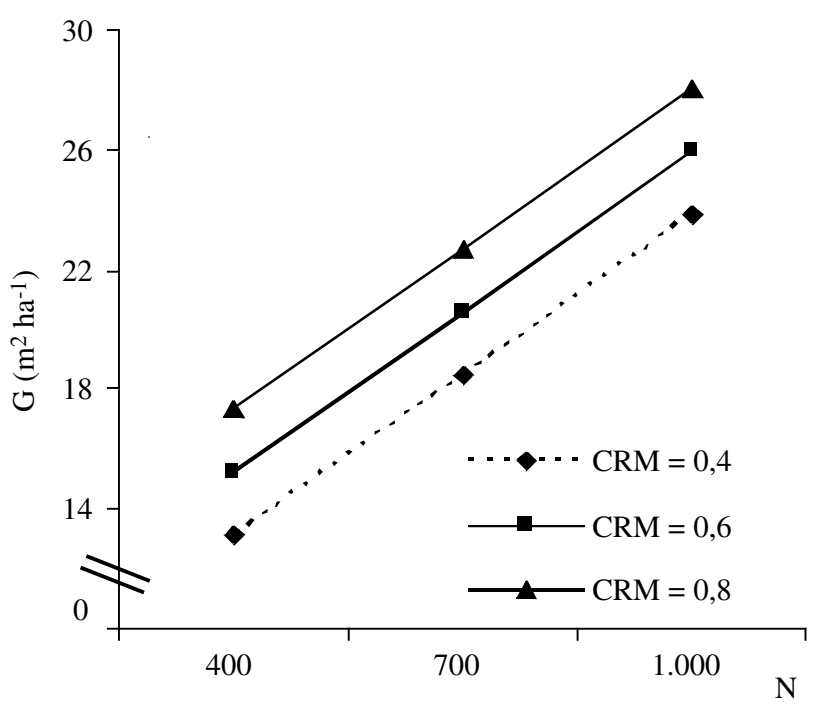

Figura 1. Área basal $(\mathrm{G})$ predicha por el modelo 6 en función del número de árboles por hectárea $(\mathrm{N})$ y de la proporción de longitud de copa promedio (CRM).

Predicted basal area $(\mathrm{G})$ by model 6 as a function of density (N) and the average crown ratio (CRM). 
Cuadro 3. Modelos predictivos del área basal y estadísticos indicadores de la calidad de los ajustes y de la capacidad de predicción de cada uno.

Adjusted predictive models for basal area and indicative statistics of adjusted quality and prediction capabilities.

\begin{tabular}{|c|c|c|c|c|}
\hline $\mathrm{N}^{\circ}$ & Modelos obtenidos ${ }^{\mathrm{a}}$ & $S_{x y}$ & $\begin{array}{c}\mathrm{R}^{2} \\
\text { ajustado }\end{array}$ & $\begin{array}{l}\text { PRESS } \\
\text { RMSE }\end{array}$ \\
\hline 1 & $\mathrm{G}=-37,7229+1,4659 \mathrm{IS}-0,0107 \mathrm{~N}+0,0040 \mathrm{EN}+0,0740 \mathrm{E}$ IS & 1,991 & 0,917 & 2,014 \\
\hline 2 & $\mathrm{G}=-40,1203+1,2561 \mathrm{IS}-0,0113 \mathrm{~N}+8,5667 \mathrm{CRA}+0,0041 \mathrm{EN}+0,0869 \mathrm{E}$ IS & 1,852 & 0,928 & 1,878 \\
\hline 3 & $\begin{array}{c}\mathrm{G}=-44,6818+1,4068 \mathrm{IS}-0,0115 \mathrm{~N}+7,4910 \mathrm{CRA}+ \\
6,0390 \mathrm{CR} 4+0,0041 \mathrm{E} \mathrm{N}+0,0759 \mathrm{E} \text { IS }\end{array}$ & 1,882 & 0,909 & 1,928 \\
\hline 4 & $\mathrm{G}=-44,4636+1,8586 \mathrm{IS}-0,0219 \mathrm{~N}+6,8106 \mathrm{CRA}+6,1025 \mathrm{CR} 4+0,0059 \mathrm{E} \mathrm{N}$ & 1,962 & 0,902 & 2,005 \\
\hline 5 & $\begin{array}{c}\mathrm{G}=-28,6628-0,0098 \mathrm{~N}+7,5414 \mathrm{CRA}+6,0488 \mathrm{CR} 4- \\
0,4282 \mathrm{E}^{2}+0,0038 \mathrm{E}+0,3082 \mathrm{E} \text { IS }\end{array}$ & 1,875 & 0,910 & 1,920 \\
\hline 6 & $\mathrm{G}=-40,4102+1,2053 \mathrm{IS}-0,0120 \mathrm{~N}+0,0043 \mathrm{E} \mathrm{N}+0,0901 \mathrm{E} \mathrm{IS}+10,5033 \mathrm{CRM}$ & 1,808 & 0,931 & 1,833 \\
\hline 7 & $\mathrm{G}=-34,4106+1,2756 \mathrm{IS}-0,0112 \mathrm{~N}-0,7019 \mathrm{HPA}+0,0041 \mathrm{E} \mathrm{N}+0,1020 \mathrm{E}$ IS & 1,885 & 0,925 & 1,911 \\
\hline 8 & $\begin{array}{c}\ln (\mathrm{G})=-11,9121+1,3902 \mathrm{E}+0,1336 \mathrm{IS}+0,5547 \mathrm{CRA}- \\
0,0854 \mathrm{E}^{2}+0,9582 \ln (\mathrm{N})-0,000065 \mathrm{E} \mathrm{N}\end{array}$ & 1,877 & 0,944 & 1,015 \\
\hline 9 & $\begin{array}{c}\ln (\mathrm{G})=-2,9174+0,1187 \mathrm{IS}+0,4719 \mathrm{CRA}+ \\
0,4017 \mathrm{CR} 4-10,9329 \mathrm{E}^{-1}+0,6453 \ln (\mathrm{N})\end{array}$ & 1,893 & 0,926 & 1,011 \\
\hline 10 & $\begin{array}{c}\ln (\mathrm{G})=-3,4684-0,3306 \mathrm{E}+0,4892 \mathrm{CRA}+0,4036 \mathrm{CR} 4- \\
25,6958 \mathrm{E}^{-2}+0,9239 \ln (\mathrm{N})-0,000055 \mathrm{E} N+0,0187 \mathrm{E} \text { IS }\end{array}$ & 1,912 & 0,924 & 1,012 \\
\hline
\end{tabular}

a $\quad \mathrm{G}=$ área basal de la masa $\left(\mathrm{m}^{2}\right.$ ha $\left.{ }^{-1}\right) ; \mathrm{E}=$ edad de la masa (años); IS = índice de sitio $(\mathrm{m}) ; \mathrm{N}$ = número de árboles por hectárea registrado por lo menos un año antes de la edad de predicción; HPA = altura de poda lograda por lo menos un año antes de la edad de predicción $(\mathrm{m}) ; \mathrm{CRA}=$ proporción de longitud de copa remanente promedio a la edad de predicción, con altura de poda lograda por lo menos un año antes de dicha edad; CR4 = proporción de longitud de copa remanente luego de las podas realizadas a los cuatro años de edad; CRM = promedio entre las proporciones de longitud de copa remanente desde la edad de cuatro años y hasta un año anterior a la edad de predicción, previas a las podas realizadas en cada oportunidad. Todos los valores de F presentaron $P<0,0001$.

Predicción del diámetro medio cuadrático. En el cuadro 4 se muestran los resultados de once modelos ajustados y algunos estadísticos indicadores de la calidad de ajuste y de la capacidad de predicción de cada uno. La función 1 fue la única que no contuvo a ninguna variable vinculada con las podas. De los ajustes de las diez funciones que contuvieron variables de poda, resultó que todas tuvieron menores $S_{y . x}$ y PRESS RMSE que la función 1 y seis tuvieron mayores $R^{2}$ ajustados que dicha función. Se destacaron las funciones identificadas con los números 8 , 9,10 y 11, que fueron las que tuvieron los menores $S_{y . x}$ y PRESS RMSE y los mayores $\mathrm{R}^{2}$ ajustados. Desde el punto de vista práctico, se destaca el modelo 11, ya que requiere del valor de HPA, posible de obtener en el terreno con pocas mediciones adicionales, y del valor de DHP4, obtenido de la diferencia entre HPA y la altura de poda promedio lograda a los cuatro años de edad (HP4), que debería estar registrada en la historia de la plantación. Como en las cuatro funciones con mejor comportamiento estadístico resultaron incluidas variables relacionadas a las podas efectuadas a los cuatro años de edad, puede deducirse que las intensidades diferentes aplicadas a esa edad temprana fueron muy importantes para explicar la variabilidad de rendimiento de Dg en años posteriores. No resultaron significativos los aportes de las variables CR5, HP5, NLP y EUP toda vez que fueron incorporadas como variables independientes en modelos ajustados no incluidos en el cuadro 4. Los modelos pre- sentados en ese cuadro que tienen entre sus variables regresoras a HP4, DHP4 y/o CR4 son utilizables desde la edad de 5 años en adelante.

$\mathrm{Al}$ probarse los resultados de las predicciones de estos modelos para una edad de siete años dentro del rango de variación de los tratamientos de poda aplicados en el ensayo, se encontró que en todos ellos el Dg fue mayor para valores altos de CR4, CRA y/o DCR4 y fue menor para valores altos de HP4, HPA y/o DHP4. En la figura 2a se muestra una aplicación del modelo 11 . Se nota muy poca sensibilidad de la ecuación 11 a las variables de poda presentes en ese modelo.

Desde el punto de vista práctico, podrían utilizarse los modelos 2 o 6 (cuadro 3) como submodelos dentro de las funciones predictivas del $\mathrm{Dg}$, inclusive el modelo 1 , que es de aplicación más sencilla que el resto. En la figura $2 \mathrm{~b}$ se muestran los valores de $\mathrm{Dg}$ predichos a una edad de 7 años por el modelo 1 , con IS $=23 \mathrm{~m}$, donde $\mathrm{G}$ está determinada por la ecuación 6 del cuadro 3. Puede observarse que por cada aumento de 0,2 unidades de CRM, se produjo un incremento del Dg de $0,89 \mathrm{~cm}$, con mayor sensibilidad de la respuesta a los tratamientos de poda aplicados, respecto del uso del modelo 11 , sin considerar el área basal determinada por la ecuación 6 del cuadro 3.

Predicción del volumen por hectárea. En el cuadro 5 se muestran los resultados de los ajustes de cinco modelos 
Cuadro 4. Modelos predictivos del diámetro medio cuadrático y estadísticos indicadores de la calidad de los ajustes y capacidad de predicción de cada uno.

Predictive models of quadratic mean diameter: indicative statistics of adjusted quality and prediction capabilities.

\begin{tabular}{|c|c|c|c|c|}
\hline $\mathrm{N}^{\mathrm{o}}$ & Modelos obtenidos ${ }^{\mathrm{a}}$ & $\mathrm{S}_{\mathrm{xy}}$ & $\begin{array}{c}\mathrm{R}^{2} \\
\text { ajustado }\end{array}$ & $\begin{array}{l}\text { PRESS } \\
\text { RMSE }\end{array}$ \\
\hline 1 & $\begin{array}{c}\mathrm{Dg}=-16,0621+6,2727 \mathrm{E}+0,2720 \mathrm{IS}+0,0081 \mathrm{~N}+ \\
0,4222 \mathrm{G}-0,2970 \mathrm{E}^{2}-0,0029 \mathrm{EN}\end{array}$ & 0,510 & 0,982 & 0,522 \\
\hline 2 & $\begin{array}{c}\mathrm{Dg}=-17,4235+6,5897 \mathrm{E}+0,2987 \mathrm{IS}+0,0079 \mathrm{~N}+ \\
0,4053 \mathrm{G}-0,1108 \mathrm{HPA}-0,3131 \mathrm{E}^{2}-0,0028 \mathrm{E} \mathrm{N}\end{array}$ & 0,501 & 0,982 & 0,514 \\
\hline 3 & $\begin{array}{c}\mathrm{Dg}=-18,4140+6,6276 \mathrm{E}+0,2913 \mathrm{IS}+0,0079 \mathrm{~N}+0,4015 \mathrm{G}+ \\
1,2667 \mathrm{CRA}-0,3208 \mathrm{E}^{2}-0,0028 \mathrm{E} \mathrm{N}\end{array}$ & 0,501 & 0,982 & 0,513 \\
\hline 4 & $\begin{array}{c}\mathrm{Dg}=23,0118+0,0534 \mathrm{E} \text { IS }-0,0033 \mathrm{E} \mathrm{N}+0,3850 \mathrm{G}- \\
72,6679 \mathrm{E}^{-1}-0,2475 \mathrm{HP} 4-0,1209 \mathrm{DHP} 4\end{array}$ & 0,487 & 0,973 & 0,501 \\
\hline 5 & $\begin{array}{c}\mathrm{Dg}=21,7411+0,0518 \mathrm{E} \text { IS }-0,0033 \mathrm{E} \mathrm{N}+0,3805 \mathrm{G}+0,0110 \mathrm{~N}- \\
72,0989 \mathrm{E}^{-1}+1,4692 \mathrm{CRA}-0,1222 \mathrm{HP} 4\end{array}$ & 0,484 & 0,973 & 0,499 \\
\hline 6 & $\begin{array}{c}\mathrm{Dg}=23,1230+0,4589 \mathrm{G}-60,3245 \mathrm{E}^{-1}+0,0062 \mathrm{~N}+ \\
132,1274 \mathrm{HD} / \mathrm{N}-0,1114 \mathrm{HP} 4-0,0628 \mathrm{DHP} 4\end{array}$ & 0,438 & 0,978 & 0,452 \\
\hline 7 & $\begin{array}{c}\mathrm{Dg}=24,3653+0,4134 \mathrm{G}-63,6201 \mathrm{E}^{-1}+0,0108 \mathrm{G} \mathrm{HPA}+ \\
0,0068 \mathrm{~N}-0,0023 \mathrm{EN}+135,8661 \mathrm{HD} / \mathrm{N}-0,3077 \mathrm{HP} 4-0,2629 \mathrm{DHP} 4\end{array}$ & 0,432 & 0,979 & 0,450 \\
\hline 8 & $\begin{array}{c}\mathrm{Dg}=9,2148+0,1709 \mathrm{IS}-0,0007 \mathrm{~N}+0,7061 \mathrm{CRA}+0,7528 \mathrm{CR} 4- \\
20,2032 \mathrm{E}^{-1}+294,9110 \mathrm{G} / \mathrm{N}\end{array}$ & 0,270 & 0,992 & 0,287 \\
\hline 9 & $\begin{aligned} \mathrm{Dg}= & 9,5829+0,1801 \mathrm{IS}-0,0007 \mathrm{~N}+0,6559 \mathrm{CRA}- \\
& 0,0940 \mathrm{HP} 4-19,5240 \mathrm{E}^{-1}+296,9410 \mathrm{G} / \mathrm{N}\end{aligned}$ & 0,274 & 0,991 & 0,290 \\
\hline 10 & $\begin{array}{c}\mathrm{Dg}=-16,8402+3,7987 \mathrm{E}+1,0046 \mathrm{IS}-0,0007 \mathrm{~N}+1,3963 \mathrm{CR} 4+ \\
299,7890 \mathrm{G} / \mathrm{N}-0,1408 \mathrm{E} \text { IS + 0,6948 DCR4 }\end{array}$ & 0,266 & 0,992 & 0,283 \\
\hline 11 & $\begin{array}{c}\mathrm{Dg}=10,0825+0,1832 \mathrm{IS}-0,0007 \mathrm{~N}-0,1401 \mathrm{HPA}-19,4704 \mathrm{E}^{-1}+ \\
298,9788 \mathrm{G} / \mathrm{N}+0,0984 \mathrm{DHP} 4\end{array}$ & 0,277 & 0,991 & 0,293 \\
\hline
\end{tabular}

a $\quad \mathrm{Dg}=$ diámetro medio cuadrático $(\mathrm{cm}) ; \mathrm{E}=$ edad de la masa (años); IS = índice de sitio $(\mathrm{m}) ; \mathrm{N}=$ número de árboles por hectárea registrado por lo menos un año antes de la edad de predicción; $\mathrm{G}=$ área basal de la masa $\left(\mathrm{m}^{2} \mathrm{ha}^{-1}\right) ; \mathrm{HPA}=$ altura de poda lograda por lo menos un año antes de la edad de predicción $(\mathrm{m}) ; \mathrm{HP} 4=$ altura de poda realizada en el $4^{\circ}$ año de edad $(\mathrm{m})$; DHP4 = diferencia entre las alturas de poda de la edad de predicción y la realizada a los cuatro años de edad (HPA - HP4); CRA = proporción de longitud de copa remanente respecto de la altura total a la edad de predicción; $\mathrm{CR} 4=$ proporción de longitud de copa remanente luego de las podas realizadas a los cuatro años de edad; DCR4= diferencia de proporciones de copa verde remanentes entre la edad de predicción y los cuatro años de edad (CRA-CR4); HD = altura dominante a la edad de predicción (m). Todos los valores de F presentaron $P<0,0001$.

lineales predictivos del volumen total sin corteza por hectárea. La función 1 fue la única que no contuvo variables vinculadas con las podas. Al ajustar esta función con variables regresoras fuertemente correlacionadas con V se obtuvieron coeficientes de determinación superiores al 99\%. Se destacó el área basal, con un coeficiente de correlación de $0,97(P<0,01)$. Como todas las variables de poda evaluadas tuvieron correlaciones más débiles con $\mathrm{V}$ que cualquiera de las variables incluidas en el modelo 1, sus incorporaciones en más de cuarenta modelos ajustados produjeron: ajustes de menor calidad que la lograda con la ecuación 1; variables estadísticamente no significativas, en especial HP5, CR5, EUP y NLP; y/o predicciones biológicamente no razonables, como el mayor volumen con el aumento de la altura de poda o con la disminución de la proporción de copa viva.
Las excepciones fueron los modelos 2 a 5 (cuadro 5). Todas las variables independientes presentes en estas funciones tuvieron contribuciones estadísticamente significativas y son utilizables para edades entre cuatro y siete años. En los análisis de residuales de estos modelos se observaron leves apartamientos de los supuestos de homogeneidad de variancia y de la distribución normal de los residuales. Del ajuste de las cuatro funciones que contuvieron variables de poda, resultó que todas tuvieron menores $S_{y . x}$ y PRESS RMSE y mayores $R^{2}$ ajustados que el modelo 1 , aunque con muy pequeñas diferencias con respecto a éste. En todos los casos el volumen predicho a los siete años de edad fue levemente superior al aumentar el CRA. Dada la escasa ganancia estadística de aplicar estos modelos, la complejidad de su aplicación (son polinomios con 10 términos como mínimo) y la escasa sensibilidad de cualquiera de ellos a la variable de 
Cuadro 5. Modelos predictivos del volumen por hectárea y estadísticos indicadores de la calidad de los ajustes y capacidad de predicción de cada uno.

Predictive models for volume per hectare and indicative statistics of adjusted quality and prediction capabilities.

\begin{tabular}{|c|c|c|c|c|}
\hline $\mathrm{N}^{\mathrm{o}}$ & Modelos obtenidos ${ }^{\mathrm{a}}$ & $S_{. x y}$ & $\mathrm{R}^{2}$ ajustado & $\begin{array}{l}\text { PRESS } \\
\text { RMSE }\end{array}$ \\
\hline 1 & $\begin{array}{c}\mathrm{V}=189,9492-53,8903 \mathrm{E}-6,0749 \mathrm{IS}-0,0249 \mathrm{~N}+4,6937 \mathrm{G}+ \\
2,3515 \mathrm{E}^{2}+0,0035 \mathrm{E} \mathrm{N}+1,3423 \mathrm{E} \mathrm{IS}\end{array}$ & 2,536 & 0,995 & 2,578 \\
\hline 2 & $\begin{array}{c}\mathrm{V}=219,3756-60,5076 \mathrm{E}-5,7192 \mathrm{IS}-0,0240 \mathrm{~N}+4,7224 \mathrm{G}-39,2263 \mathrm{CRA}+ \\
2,7111 \mathrm{E}^{2}+0,0033 \mathrm{E}+1,2675 \mathrm{E} \mathrm{IS}+6,3223 \mathrm{E} \mathrm{CRA}\end{array}$ & 2,500 & 0,995 & 2,552 \\
\hline 3 & $\begin{array}{c}V=314,3267-51,3758 \mathrm{E}-5,5996 \mathrm{IS}-0,0246 \mathrm{~N}+4,7226 \mathrm{G}+2,9540 \mathrm{E}^{2}- \\
285,7486 \mathrm{CRA}+0,0034 \mathrm{E}+1,2338 \mathrm{E} \mathrm{IS}+166,1012 \mathrm{CRA}^{2}-4,5925 \mathrm{E} / \mathrm{CRA}\end{array}$ & 2,442 & 0,996 & 2,499 \\
\hline 4 & $\begin{array}{c}\mathrm{V}=212,5918-60,1516 \mathrm{E}-5,5749 \mathrm{IS}-0,0228 \mathrm{~N}+5,1051 \mathrm{G}-39,4605 \mathrm{CRA}+ \\
2,6451 \mathrm{E}^{2}-0,6127 \mathrm{G} \mathrm{CRA}+0,0031 \mathrm{E}+1,2459 \mathrm{E} \mathrm{IS}+8,1622 \mathrm{E} \text { CRA }\end{array}$ & 2,488 & 0,996 & 2,547 \\
\hline 5 & $\begin{array}{c}V=-289,7573-5,9339 \mathrm{IS}-0,0239 \mathrm{~N}+4,7196 \mathrm{G}+1.883,6048 \mathrm{E}^{-1}- \\
38,2796 \mathrm{CRA}-2.551,2882 \mathrm{E}^{-2}+0,0033 \mathrm{EN}+1,3077 \mathrm{E} \mathrm{IS}+6,1668 \mathrm{E} \mathrm{CRA}\end{array}$ & 2,499 & 0,995 & 2,551 \\
\hline
\end{tabular}

a $\quad \mathrm{V}=$ volumen total sin corteza de la masa $\left(\mathrm{m}^{3} \mathrm{ha}^{-1}\right) ; \mathrm{E}=$ edad de la masa (años); IS = índice de sitio $(\mathrm{m}) ; \mathrm{N}=$ número de árboles por hectárea registrado por lo menos un año antes de la edad de predicción; $\mathrm{G}=$ área basal de la masa $\left(\mathrm{m}^{2} \mathrm{ha}^{-1}\right)$; $\mathrm{CRA}=$ proporción de longitud de copa remanente promedio a la edad de predicción, con altura de poda lograda por lo menos un año antes de dicha edad. Todos los valores de F presentaron $P<0,0001$

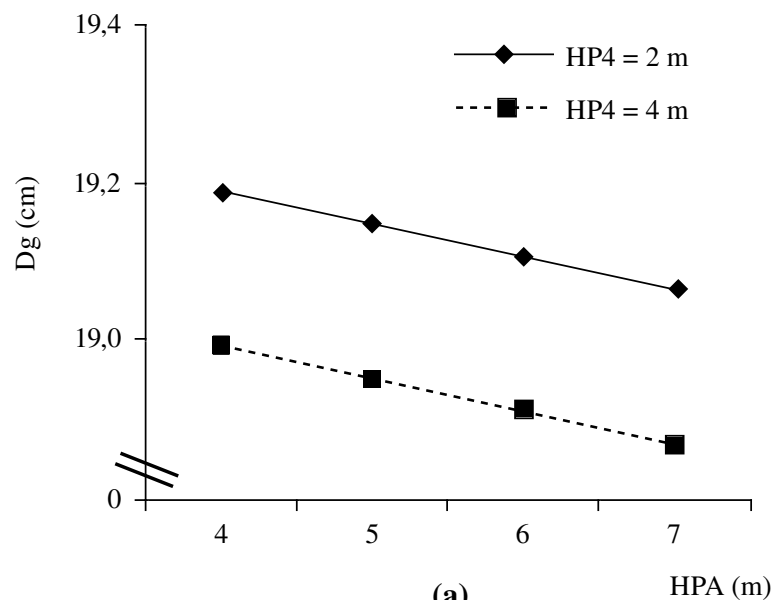

(a)

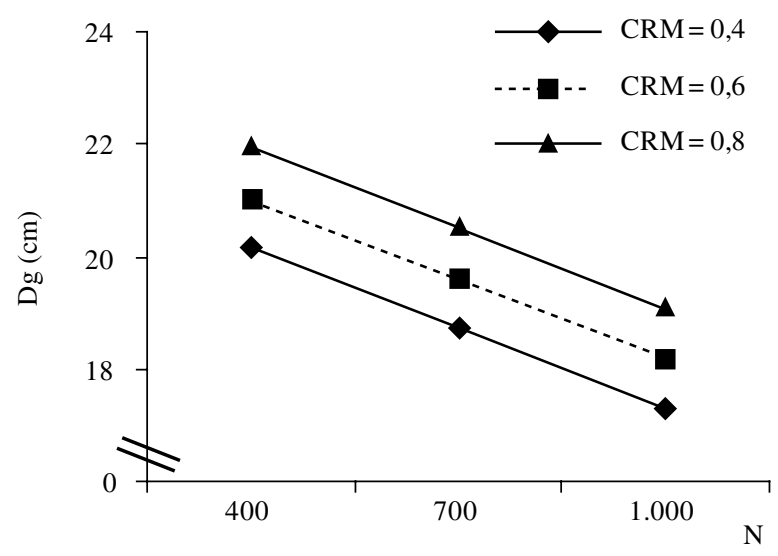

(b)

Figura 2. (a) Diámetro medio cuadrático (Dg) predicho por el modelo 11 en función de la altura de poda actual (HPA) y la altura de poda lograda a la edad de 4 años (HP4). (b) Dg predicho con el modelo 1, utilizando el G calculado con la función 6 del cuadro 3.

(a) Predicted quadratic mean diameter (Dg) for model 11 as a function of present prune height (HPA) and the prune height at the age 4 (HP4). (b) Predicted Dg with model 1, employing the function number 6 of table 3 for basal area.

poda presente, no resultan prácticos ni eficientes para predecir el volumen de masas juveniles podadas. Para este fin se podría utilizar la función 1, con muy buen comportamiento estadístico, utilizando los modelos 2 o 6 del cuadro 3 para obtener el área basal, como submodelos dentro de la función predictiva del volumen. En la figura 3 se muestran los valores de $\mathrm{V}$ predichos a una edad de 7 años por el modelo 1 , con IS $=23 \mathrm{~m}$, donde $\mathrm{G}$ está determinada por el modelo 6 del cuadro 3. Puede observarse que por cada incremento de 0,2 unidades de
CRM, se produjo un aumento del volumen de $9,86 \mathrm{~m}^{3}$ $\mathrm{ha}^{-1}$, resultado biológicamente razonable acorde a lo recomendado por Huang et al. (2003).

\section{DISCUSIÓN Y CONCLUSIONES}

Se obtuvieron funciones estáticas de rodal completo que permiten cuantificar la influencia de la intensidad y la oportunidad de las podas sobre el rendimiento en eda- 


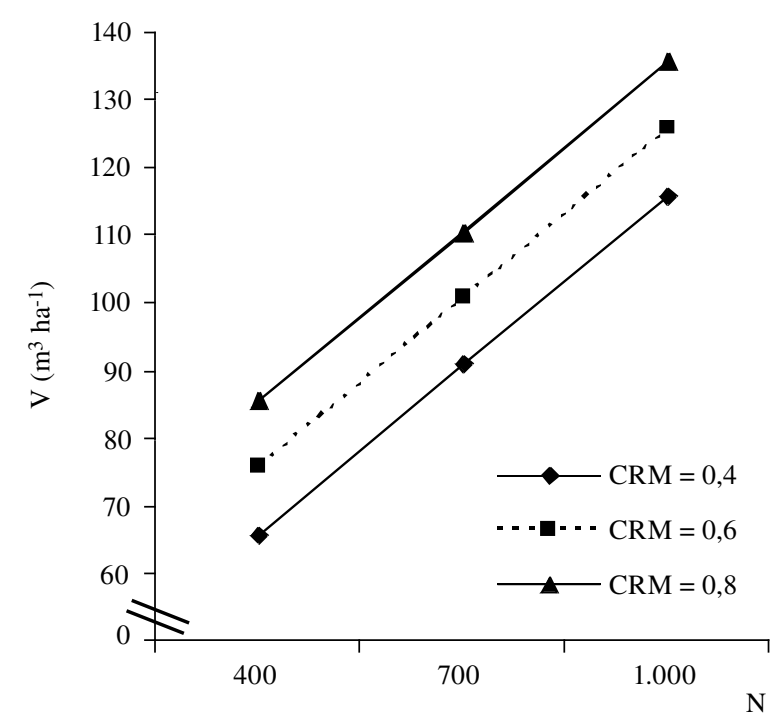

Figura 3. Volumen por hectárea (V) predicho con el modelo 1, utilizando el área basal calculada con la función 6 del cuadro 3 .

Estimated volume by hectare with model 1, using the basal area obtained with function 6 of table 3 .

des juveniles, utilizables como herramientas de cálculo para decidir cuánto y cuándo podar ramas fotosintéticamente activas de acuerdo a los objetivos de producción.

$\mathrm{Al}$ incorporar variables vinculadas con las podas en modelos predictivos de área basal se obtienen modelos de mejor calidad de ajuste y capacidad de predicción que funciones con excelentes comportamientos estadísticos, sin variables de poda. La utilización de algunas de esas ecuaciones como submodelos, dentro de funciones predictivas de diámetro medio cuadrático y volumen por hectárea, relativamente sencillos y con confiabilidad estadística, producen resultados sensibles a las podas. Las predicciones efectuadas con los modelos predictivos del área basal tienen el mismo sentido que los resultados de las comparaciones de tratamientos de poda mostrados por Costas et al. (2005), realizadas con este mismo ensayo a los siete años de edad. Se verifica que todas las funciones obtenidas resultan ser biológicamente razonables, acorde a lo recomendado por Huang et al. (2003).

El efecto de la proporción de longitud de copa remanente sobre el diámetro medio es similar al mostrado por las funciones predictivas del diámetro a la altura de pecho de cada árbol obtenidas por Burkhart el al. (2001, 2003), construidas con datos de plantaciones de edades entre 8 y 25 años. Aunque en el ensayo no se aplicaron podas severas $(\mathrm{CR}>0,35)$, los modelos generados en este trabajo permiten predecir para edades de plantación juveniles la menor producción en diámetro medio que Meneses y Guzmán (2000) describen que ocurre hasta la edad de cosecha en caso de podas muy severas.

Las funciones obtenidas son promisorias para la investigación sobre modelos y sistemas locales de simula- ción de crecimiento y producción de $P$. taeda que contemplen a las podas realizadas en los sistemas de producción intensivos.

\section{AGRADECIMIENTOS}

A los alumnos y exalumnos de Ingeniería Forestal de la Facultad de Ciencias Forestales: Mariano Cáceres, Ramón Silva, Rosa Winck, Liliana Rivero, Eusebio Winck, Reinaldo Jara, Javier Giménez, Graciela Martínez, Omar Martínez, Martín Vargas, Raquel Winck, Rafaela Morales, Juan Heck, Iván Kahlstorf y Marisa Becker por su participación ad honorem en algunos de los cuatro años de medición del ensayo.

Al personal de campo de la empresa del ingeniero Pedro Irschick por su colaboración en la aplicación de los tratamientos del ensayo y en la recolección de datos.

\section{REFERENCIAS}

Alder D. 1980. Estimación del Volumen Forestal y Predicción del Rendimiento. Vol. 2: Predicción del Rendimiento. Estudio FAO: Montes 22/2. Roma, Italia. Organización de las Naciones Unidas para la Agricultura y la Alimentación. 200 p.

Avery TE, H Burkhart. 1994. Forest measurements. New York, USA. McGraw-Hill. $4^{\mathrm{a}}$ Edition. 408 p.

Burkhart HE, KD Farrar, RL Amateis, RF Daniels. 2001. Simulation of individual tree growth and stand development in loblolly pine plantations on cutover, site-prepared areas. Publication $N^{\circ}$ FWS-1-87. Blacksburg, Virginia 24061, USA. Department of Forestry, Virginia Tech. 22 p.

Burkhart HE, RL Amateis, JA Westfall, RF Daniels. 2003. PTAEDA3: Simulation of individual tree growth, stand development and economic evaluation in loblolly pine plantations. Blacksburg, Virginia 24061, USA. Department of forestry, Virginia Tech. $23 \mathrm{p}$.

Costas RA, P Mac Donagh, E Weber, S Figueredo, P Irschick. 2005. Influencias de la densidad y podas sobre la producción de Pinus taeda L. a los 7 años de edad. Ciencia Florestal 15(3):275-284.

Crechi EH, HE Fassola, RA Friedl. 1998. Simulador Forestal. Pinus elliottii V 1.0. Manual de descripción técnica. Programa Cooperativo Simulador de Crecimiento INTA-FCF. Montecarlo, Misiones, Argentina. 18 p.

Crechi EH, HE Fassola, P Ferrere. 1999. Simulador Forestal. Pinus taeda V 2.0. Manual de descripción técnica. Programa Cooperativo Simulador de Crecimiento INTA. Montecarlo, Misiones, Argentina. 16 p.

Evans J. 1992. Plantation Forestry in the tropics. New York. USA. Oxford University Press. Second Edition. 403 p.

Huang S, Y Yang, Y Wang. 2003. A critical look at procedures for validating growth and yield models. In Amaro A, D Reed, A Soares eds. Modelling Forest Systems. Instituto Superior de Gestao, Lisbon, Portugal. Michigan Technological University, USA. Instituto Superior de Agronomia, Lisbon, Portugal. p. 271-293. 
Meneses M, S Guzmán. 2000. Análisis de la eficiencia de la silvicultura destinada a la obtención de madera libre de nudos en plantaciones de pino radiata en Chile. Bosque 21(2):85-93.

Muñoz F, M Espinosa, MA Herrera, J Cancino. 2005. Características del crecimiento en diámetro, altura y volumen de una plantación de Eucalyptus nitens sometida a tratamientos silvícolas de poda y raleo. Bosque 26(1):93-99.

Myers RH. 1990. Classical and Modern Regression with Applications. Boston, USA. PWS-KENT Publishing Company. $488 \mathrm{p}$.
Prodan M, R Peters, F Cox, P Real. 1997. Mensura Forestal. San José, Costa Rica. IICA-BMZ/GTZ. 561 p.

Sanquetta CR. 1996. Fundamentos biométricos dos modelos de simulaçao florestal. Curitiba, Brasil. FUPEF. 49 p. (Serie Didáctica $\left.\mathrm{N}^{\circ} 08\right)$.

Seitz RA. 1995. Manual da Poda de Espécies Arbóreas Florestais. Curitiba, Brasil. FUPEF. 88 p.

Soil Survey Staff. 1991. Keys to Soil Taxonomy. Washignton DC, USA. USDA Soil Survey Staff, Soil Conservation Service. $754 \mathrm{p}$.

Recibido: 31.05 .05

Aceptado: 14.06.06 